\title{
Bijective Interval-Valued Fuzzy Soft Set with Its Operations
} Ke Gong ${ }^{1, a}$, Panpan Wang ${ }^{1, b}$, Zhihui $Q i^{1, c}$

\author{
${ }^{1}$ School of Management, Chongqing Jiaotong University, Chongqing 400074, PR China
}

agks_cn@163.com, b874429863@qq.com, ${ }^{\text {c } 449253988 @ q q . c o m}$

Keywords:bijective interval-valued fuzzy soft set; soft set; interval-valued fuzzy soft set

\begin{abstract}
Bijective soft set is a valid approach for data mining and data analysis. This paper extends the notion to interval-valued fuzzy soft set, which can deal with more uncertain problems. The concept of bijective interval-valued fuzzy soft set is introduced and in the meantime, some of its operations and properties are discussed and studied.
\end{abstract}

\section{Introduction}

Soft set theory, proposed by Molodtsov [1], is a newly-emerging tool to deal with uncertainties. In recent years, research on soft set theory has been active, and great progress has been achieved, including the works of the using of fundamental soft set theory and soft set theory for data analysis under incomplete information [2], decision making problems [3,4], normal parameter reduction [5].

However, in real life many problems are imprecise in nature. Classical soft set theory is not capable of successfully dealing with such problems. The purpose of this paper is to combine bijective soft sets [6] and interval-valued fuzzy sets [7], which generates an alternative approach for precise data mining in interval-valued fuzzy environments.

\section{Preliminary}

\section{Soft sets}

Let $U$ be a common universe and let $E$ be a set of parameters.

Definition 1 [1] A pair $(F, E)$ is called a soft set (over $U$ ) if and only if $F$ is a mapping of $E$ into the set of all subsets of the set $U$, where $F$ is a mapping given by $F: E \rightarrow P(U)$.

In other words, the soft set is a parameterized family of subsets of the set $U$. Every set $F(\varepsilon)(\varepsilon \in E)$, from this family may be considered the set of $\varepsilon$-elements of the soft sets $(F, E)$, or as the set of $\varepsilon$-approximate elements of the soft set.

Example 1 Let universe $U=\left\{h_{1}, h_{2}, h_{3}, h_{4}\right\}$ be a set of houses, a set of parameters $E=\left\{e_{1}, e_{2}, e_{3}, e_{4}\right\}$ be a set of status of houses which stand for the parameters "beautiful", "cheap", "in green surroundings", and "in good location" respectively. Consider the mapping $F$ be a mapping of $E$ into the set of all subsets of the set $U$. Now consider a soft set $(F, E)$ that describes the "attractiveness of houses for purchase". According to the data collected, the soft set $(F, E)$ is given by

$$
\begin{aligned}
(F, E)= & \left\{\left(e_{1},\left\{h_{1}, h_{3}, h_{4}\right\}\right),\left(e_{2},\left\{h_{1}, h_{2}\right\}\right),\right. \\
& \left.\left(e_{3},\left\{h_{1}, h_{3}\right\}\right),\left(e_{4},\left\{h_{2}, h_{3}, h_{4}\right\}\right)\right\}
\end{aligned}
$$

where $F\left(e_{1}\right)=\left\{h_{1}, h_{3}, h_{4}\right\}, F\left(e_{2}\right)=\left\{h_{1}, h_{2}\right\} F\left(e_{3}\right)=\left\{h_{1}, h_{3}\right\}$ and $F\left(e_{4}\right)=\left\{h_{2}, h_{3}, h_{4}\right\}$.

\section{Interval-valued fuzzy soft sets}

Let $U$ be a common universe and let $E$ be a set of parameters.

Definition 2(see[8] ) Suppose $A \subset E$. Let $\tilde{\wp}(U)$ be the set of all fuzzy subsets in $U$. A pair $(\tilde{\wp}, A)$ is called a fuzzy soft set over $U$, where $\tilde{F}$ is a mapping given by $\tilde{F}: A \rightarrow \tilde{\wp}(U)$.

Definition 3 [7] A pair $(\tilde{\wp}, E)$ is called an interval-valued fuzzy soft set over $\tilde{\zeta}(U)$, where $\tilde{\wp}$ is a mapping given by $\tilde{\wp}: E \rightarrow \tilde{\zeta}(U)$. 
Suppose that $\tilde{\wp}(e) \in \tilde{\zeta}(U), \forall x \in U, \mu_{\tilde{\xi}(e)}(x)=\left[\mu_{\tilde{\wp}(e)}^{-}(x), \mu_{\tilde{\xi}(e)}^{+}(x)\right]$ is called the degree of membership an element $x$ to $\tilde{\wp}(e) \cdot \mu_{\tilde{\wp}(e)}^{-}(x), \mu_{\tilde{\wp}(e)}^{+}(x)$ are the lower and upper degrees of membership $x$ to $\tilde{\wp}(e)$ where $0 \leq \mu_{\tilde{\rho}(e)}^{-}(x) \leq \mu_{\tilde{\xi}(e)}^{+}(x) \leq 1$.

\section{Bijective soft sets}

Definition 4(see [6]) Let $(F, B)$ be a soft set over a common universe $U$, where $F$ is a mapping $F: B \rightarrow P(U)$ and $B$ is nonempty parameter set. We say that $(F, B)$ is a bijective soft set, if $(F, B)$ such that

(i) $\bigcup_{e \in B} F(e)=U$.

(ii) For any two parameters $e_{i}, e_{j} \in B, e_{i} \neq e_{j}, F\left(e_{i}\right) \cap F\left(e_{j}\right)=\varnothing$.

To illustrate this definition, we gave an example below.

Example 2 Suppose that $U=\left\{x_{1}, x_{2}, x_{3}, x_{4}, x_{5}, x_{6}, x_{7}\right\}$ is a common universe, $(F, E)$ is a soft set over $U, E=\left\{e_{1}, e_{2}, e_{3}, e_{4}\right\}$. The mapping of $(F, E)$ is given below: $F\left(e_{1}\right)=\left\{x_{1}, x_{2}, x_{3}\right\}, F\left(e_{2}\right)=\left\{x_{4}, x_{5}, x_{6}\right\}$, $F\left(e_{3}\right)=\left\{x_{7}\right\}, F\left(e_{4}\right)=\left\{x_{4}, x_{5}, x_{6}, x_{7}\right\}$.From Definition 4, $\left(F,\left\{e_{1}, e_{2}, e_{3}\right\}\right)$ and $\left(F,\left\{e_{1}, e_{4}\right\}\right)$ are bijective soft sets. While $\left(F,\left\{e_{1}, e_{2}\right\}\right)$ and $\left(F,\left\{e_{1}, e_{3}\right\}\right)$ are not bijective soft sets.

\section{Bijective interval-valued fuzzy soft sets and its properties}

To formulate the concept of bijective interval-valued fuzzy soft set, we will give an example below firstly. And it will be used to illustrate some notions of this section.

Example 3 Let $U=\left\{x_{1}, x_{2}, x_{3}, x_{4}, x_{5}, x_{6}\right\}$ be a common universe, is a set of six stores. Suppose that the six stores are characterized by a interval-valued fuzzy soft set $(\tilde{\wp}, E)$ over a common universe $U$. $E$ denotes the parameter set, $E=E_{1} \cup E_{2} \cup E_{3} \cup E_{4}$. $E_{1}$ describes the empowerment of sales personnel. $E_{2}$ describes the perceived quality of merchandise. $E_{3}$ describes the high traffic location. And $E_{4}$ describes store profit or loss. The sets of these parameters are $E_{1}=\{$ high, med., low $\}, E_{2}$ $=\{$ good, avg. $\}, E_{3}=\{$ no, yes $\}$ and $E_{4}=\{$ profit, loss $\}$, respectively. And $\left(\tilde{\wp}_{i}, E_{i}\right)$ is soft subset of $(\tilde{\wp}, E)$, where $i=1,2,3,4$. The mappings of two interval-valued fuzzy soft sets over $U$ are shown in Table 1 and Table 2.

Table 1 The interval-valued fuzzy soft set $\left(\tilde{\wp}_{1}, E_{1}\right)$

\begin{tabular}{llll}
\hline & high & med & low \\
\hline$x_{1}$ & {$[0.7,0.8]$} & {$[0.2,0.4]$} & {$[0.2,0.4]$} \\
$x_{2}$ & {$[0.5,0.6]$} & {$[0.7,0.9]$} & {$[0.6,0.8]$} \\
$x_{3}$ & {$[0.5,0.7]$} & {$[0.8,0.9]$} & {$[0.5,0.7]$} \\
$x_{4}$ & {$[0.2,0.4]$} & {$[0.5,0.6]$} & {$[0.7,0.9]$} \\
$x_{5}$ & {$[0.6,0.8]$} & {$[0.7,0.9]$} & {$[0.5,0.6]$} \\
$x_{6}$ & {$[0.7,0.9]$} & {$[0.5,0.6]$} & {$[0.2,0.3]$} \\
\hline
\end{tabular}

Table 2 The interval-valued fuzzy soft set $\left(\tilde{\wp}_{4}, E_{4}\right)$

\begin{tabular}{lll}
\hline & profit & loss \\
\hline$x_{1}$ & {$[0.8,1.0]$} & {$[0.2,0.4]$} \\
$x_{2}$ & {$[0.5,0.6]$} & {$[0.7,0.9]$} \\
$x_{3}$ & {$[0.8,0.9]$} & {$[0.5,0.7]$} \\
$x_{4}$ & {$[0.2,0.3]$} & {$[0.8,0.9]$} \\
$x_{5}$ & {$[0.5,0.7]$} & {$[0.8,0.9]$} \\
$x_{6}$ & {$[0.7,0.9]$} & {$[0.5,0.7]$} \\
\hline
\end{tabular}




\section{Concept of bijective interval-valued fuzzy soft set}

Definition 5 Let $(\tilde{\wp}, E)$ be an interval-valued fuzzy soft set over universe $\tilde{\zeta}(U), x$ be an element of $U$ and $e$ be a parameter of $E$. Suppose that $\tilde{\wp}(e) \in \tilde{\zeta}(U), \forall x \in U, \mu_{\tilde{\xi}(e)}(x)=\left[\mu_{\tilde{\xi}(e)}^{-}(x), \mu_{\tilde{\xi}(e)}^{+}(x)\right]$ is called the degree of membership an element $x$ to $\tilde{\wp}(e) \cdot \mu_{\tilde{\xi}(e)}^{-}(x), \mu_{\tilde{\xi}(e)}^{+}(x)$ are the lower and upper degrees of membership $x$ to $\tilde{\wp}(e)$ where $0 \leq \mu_{\tilde{\wp}(e)}^{-}(x) \leq \mu_{\tilde{\rho}(e)}^{+}(x) \leq 1 . \xi_{x}^{\left[t_{1}, t_{2}\right]}(e)$ denotes the characteristic function of the $\left[t_{1}, t_{2}\right]$-level of the interval-valued fuzzy soft set $(\tilde{\wp}, E)$, defined by

$$
\xi_{x}^{\left[t_{1}, t_{2}\right]}(e)=\left\{\begin{array}{cc}
1 & \text { if } t_{1} \leq \mu_{\tilde{\wp}(e)}^{-}(x), \mu_{\tilde{\wp}(e)}^{+}(x) \geq t_{2} \\
0 & \text { else }
\end{array}\right.
$$

where $0 \leq t_{1} \leq t_{2} \leq 1$.

Example 4 Consider Example 3, suppose that we obtain the $\left[t_{1}, t_{2}\right]$-level soft set $(F, E)$ of the interval-valued fuzzy soft $\operatorname{set}(\tilde{\wp}, E)$ as follows:

$$
\begin{array}{lc}
F_{1}(\text { high })=\left\{1 / x_{1}, 0 / x_{2}, 0 / x_{3}, 0 / x_{4}, 0 / x_{5}, 1 / x_{6}\right\}, & F_{1}(\text { med. })=\left\{0 / x_{1}, 1 / x_{2}, 1 / x_{3}, 0 / x_{4}, 1 / x_{5}, 0 / x_{6}\right\}, \\
F_{1}(\text { low })=\left\{0 / x_{1}, 0 / x_{2}, 0 / x_{3}, 1 / x_{4}, 0 / x_{5}, 0 / x_{6}\right\}, & F_{2}(\text { good })=\left\{1 / x_{1}, 1 / x_{2}, 1 / x_{3}, 0 / x_{4}, 0 / x_{5}, 0 / x_{6}\right\}, \\
F_{2}(\text { avg. })=\left\{0 / x_{1}, 0 / x_{2}, 0 / x_{3}, 1 / x_{4}, 1 / x_{5}, 1 / x_{6}\right\}, & F_{3}(\text { no })=\left\{1 / x_{1}, 1 / x_{2}, 1 / x_{3}, 1 / x_{4}, 0 / x_{5}, 0 / x_{6}\right\}, \\
F_{3}(\text { yes })=\left\{0 / x_{1}, 0 / x_{2}, 0 / x_{3}, 0 / x_{4}, 1 / x_{5}, 1 / x_{6}\right\}, & F_{4} \text { (profit) }=\left\{1 / x_{1}, 0 / x_{2}, 1 / x_{3}, 0 / x_{4}, 0 / x_{5}, 1 / x_{6}\right\}, \\
F_{4}(\text { loss })=\left\{0 / x_{1}, 1 / x_{2}, 0 / x_{3}, 1 / x_{4}, 1 / x_{5}, 0 / x_{6}\right\}, &
\end{array}
$$

where $\left[t_{1}, t_{2}\right]$-level equals to $[0.7,0.8]$.

Definition 6 Let $(F, B)$ be the $\left[t_{1}, t_{2}\right]$-level soft set of a interval-valued fuzzy $\operatorname{soft} \operatorname{set}(\tilde{\wp}, B)$ over a common universe $U, B$ is a nonempty parameter set. We say that $(\tilde{\wp}, B)$ is a $\left[t_{1}, t_{2}\right]$-level bijective interval-valued fuzzy soft set, if and only if $(F, B)$ is a bijective soft set.

Example 5 Let us reconsider the interval-valued fuzzy soft sets given in Example 4. From Definition 6

Definition 6, $\left(\tilde{\wp}_{i}, E_{i}\right) \quad i=1,2,3,4$, are bijective interval-valued fuzzy soft sets respectively.

Theorem 1 Suppose that $(\tilde{\wp}, A)$ and $(\tilde{\zeta}, B)$ are two $\left[t_{1}, t_{2}\right]$-level bijective interval-valued fuzzy soft sets over common universe $U \cdot(\tilde{\varsigma}, C)=(\tilde{\wp}, A) \wedge(\tilde{\zeta}, B)$ is a $\left[t_{1}, t_{2}\right]$-level bijective interval-valued fuzzy soft set.

Theorem 2 Suppose that $(\tilde{\wp}, A)$ is a $\left[t_{1}, t_{2}\right]$-level bijective interval-valued fuzzy soft set over $U$ and $(\tilde{\zeta}, B)$ is a Null soft set over $U \cdot(\tilde{\varsigma}, C)=(\tilde{\wp}, A) \tilde{U}(\tilde{\zeta}, B)$ is a $\left[t_{1}, t_{2}\right]$-level bijective interval-valued fuzzy soft set.

\section{Some operations on bijective interval-valued fuzzy soft sets}

Definition 7 The mean bijective interval-valued fuzzy soft set of the $\left[t_{1}, t_{2}\right]$-level bijective interval-valued fuzzy soft set $(\tilde{\wp}, E)$ is defined as a fuzzy soft set $\left(\tilde{\wp}_{m}, E\right)$ over $U$ such that

$$
\tilde{\wp}_{m}(e)=\left\{\left(x,\left(\mu_{\tilde{\wp}(e)}^{-}(x)+\mu_{\tilde{\wp}(e)}^{+}(x)\right) / 2\right): x \in U\right\}, \forall e \in E
$$

Denote $v_{m}(x)=\frac{\mu_{\tilde{\rho}(e)}^{-}(x)+\mu_{\tilde{\rho}(e)}^{+}(x)}{2}, \forall e \in E \quad x \in U$

Here, $\forall x \in U, \mu_{\tilde{\wp}(e)}^{-}(x), \mu_{\tilde{\wp}(e)}^{+}(x)$ are the lower and upper degrees of membership of $x$ to $\tilde{\wp}(e)$, $0 \leq \mu_{\tilde{\xi}(e)}^{-}(x) \leq \mu_{\tilde{\wp}(e)}^{+}(x) \leq 1$.

Definition 8 Suppose that $(\tilde{\wp}, E)$ is a $\left[t_{1}, t_{2}\right]$-level bijective interval-valued fuzzy soft set over $\tilde{\zeta}(U)$ ,$\left(\tilde{\wp}_{m}, E\right)$ is the mean bijective interval-valued fuzzy soft set of $(\tilde{\wp}, E)$, and $(F, E)$ is a $\left[t_{1}, t_{2}\right]$-level soft set of $(\tilde{\wp}, E)$, a product of $\left(\tilde{\wp}_{m}, E\right)$ and $(F, E)$ is called the combined $\left[t_{1}, t_{2}\right]$-level bijective intervalvalued fuzzy soft set of $(\tilde{\wp}, E)$, denoted by $(\wp, E)$ and defined by 


$$
\wp(e)_{\left[t_{1}, t_{2}\right]}=\left\{\begin{array}{cl}
\tilde{\wp}_{m}(e) & \text { if } \xi_{x}^{\left[t_{1}, t_{2}\right]}(e)=1 \\
0 & \text { if } \xi_{x}^{\left[t_{1}, t_{2}\right]}(e)=0
\end{array}, \forall e \in E, x \in U\right.
$$

Definition 9 Suppose that $(\tilde{\wp}, E)$ is a $\left[t_{1}, t_{2}\right]$-level bijective interval-valued fuzzy soft set over $\tilde{\zeta}(U) \cdot(\wp, E)$ is a combined $\left[t_{1}, t_{2}\right]$-level bijective interval-valued fuzzy soft set of $(\tilde{\wp}, E)$. Then the total membership degree of $(\wp, E)$ can be defined as:

$$
\tilde{T}(e)_{\left[t_{1}, t_{2}\right]}=\sum_{x \in \wp(e)} v_{m}(x)_{\left[t_{1}, t_{2}\right]}, \text { for all } e \in E .
$$

Here, ${ }_{v_{m}}(x)=\frac{\mu_{\tilde{\hat{\sigma}}(e)}(x)+\mu_{\hat{\phi}^{\dagger}(e)}(x)}{2}, \forall e \in E x \in U$.

Definition 10 (restricted AND operation on a bijective interval-valued fuzzy soft set and a subset of universe) Let $U=\left\{x_{1}, x_{2}, \ldots, x_{n}\right\}$ be a common universe, and $\tilde{\zeta}(U)$ be the set of all fuzzy subsets in $U . \tilde{X}$ be a fuzzy subset of $U$, and $(\tilde{\wp}, E)$ be a $\left[t_{1}, t_{2}\right]$-level bijective interval-valued fuzzy soft set over $\tilde{\zeta}(U)$. The operation of “ $(\tilde{\wp}, E)$ restricted $A N D \tilde{X}$ ” denoted by $(\tilde{\wp}, E) \wedge \tilde{X}$ is defined by $\bigcup_{e \in E}\left\{\wp(e)_{\left[t_{1}, t_{2}\right]}: \wp(e)_{\left[t_{1}, t_{2}\right]} \tilde{\subset} \tilde{X}\right\}$.

Here, $\wp(e)_{\left[t_{1}, t_{2}\right]}=\left\{\begin{array}{cc}\tilde{\xi}_{m}(e) & \text { if } \xi_{x}^{\left[t_{t}, t_{2}\right]}(e)=1 \\ 0 & \text { if } \xi_{x}^{\left[t_{1}, 2\right]}(e)=0\end{array}, \forall e \in E, x \in U\right.$

Example 6 Let $(\tilde{\wp}, A)$ be a $\left[t_{1}, t_{2}\right]$-level bijective interval-valued fuzzy soft set over $\tilde{\zeta}(U)$ $\tilde{\zeta}(U)=\left\{0.90 / x_{1}, 0.85 / x_{2}, 0.90 / x_{3}, 0.90 / x_{4}\right\}$. Suppose the following:

$\left(\wp_{3} A\right)=\left\{e_{1}\left\{0.90 / x_{1}\right\}, e_{2}\left\{0.85 / x_{2}, 0.90 / x_{3}\right\}, e_{3}\left\{0.90 / x_{4}\right\}\right\} \quad \tilde{X}=\left\{0.90 / x_{2}, 90 / x_{3}\right\}$

From Definition 10, we can write $(\tilde{\wp}, A) \wedge \tilde{X}=\left\{0.85 / x_{2}, 0.90 / x_{3}\right\}$.

Dependency between two bijective interval-valued fuzzy soft sets

Definition 11 (dependency between two bijective interval-valued fuzzy soft sets) Suppose that $(\tilde{\wp}, A),(\tilde{\zeta}, C)$ are two $\left[t_{1}, t_{2}\right]$-level bijective interval-valued fuzzy soft sets over a common universe $U$, where $A \cap C=\varnothing . \quad(\varsigma, P)=\bigcup_{e \in C}(\tilde{\wp}, A) \wedge \tilde{\zeta}(e) \cdot(\tilde{\wp}, A)$ is said to depend on $(\tilde{\zeta}, C)$ to a degree $k_{\left[t_{1}, t_{2}\right]}\left(0 \leq k_{\left[t_{1}, t_{2}\right]} \leq 1\right)$, denoted $(\tilde{\wp}, A) \Rightarrow_{k}(\tilde{\zeta}, C)$, if

$$
k_{\left[t_{1}, t_{2}\right]}=\gamma_{\left[t_{1}, t_{2}\right]}((\tilde{\wp}, A),(\tilde{\zeta}, C))=\left(\frac{\tilde{T}(p)}{\tilde{T}(a)}\right)_{\left[t_{1}, t_{2}\right]}
$$

where $\tilde{T}(a)_{\left[t_{1}, t_{2}\right]}=\sum_{x \in \wp(a)} v_{m}(x)_{\left[t_{1}, t_{2}\right]}$

$v_{m}(x)=\frac{\mu_{\tilde{\rho}(e)}^{-}(x)+\mu_{\tilde{\rho}(e)}^{+}(x)}{2}$,

$\tilde{T}(p)_{\left[t_{1}, t_{2}\right]}=\sum_{x \in \varsigma(p)} v_{m}(x)_{\left[t_{1}, t_{2}\right]}$ ( see Definition 9).

The concept of dependency is to describe a degree of bijective interval-valued fuzzy soft set in classifying the other one.

If $k_{\left[t_{1}, t_{2}\right]}=1$ we say $(\tilde{\wp}, A)$ is full depended on $(\tilde{\zeta}, C)$.

If $k_{\left[t_{1}, t_{2}\right]}=0$ we say $(\tilde{\wp}, A)$ is not depended on $(\tilde{\zeta}, C)$.

Example 7 Let us reconsider the $\left[t_{1}, t_{2}\right]$-level bijective interval-valued fuzzy soft sets given in Example 4. From Definition 11, we can get $k_{[0.7,0.8]}=\gamma_{[0.7,0.8]}\left(\left(\tilde{\wp}_{1}, E_{1}\right),\left(\tilde{\wp}_{4}, E_{4}\right)\right)=0.4895$. 


\section{Bijective interval-valued fuzzy soft decision system and its reduction sets}

Definition 12 (bijective interval-valued fuzzy soft decision system) Let set $U=\left\{x_{1}, x_{2}, \ldots, x_{n}\right\}$ be a common universe, and $\tilde{\zeta}(U)$ be the set of all fuzzy subsets in $U$.Suppose that $\left(\tilde{\wp}_{i}, E_{i}\right)(i=1,2,3, \ldots, n)$

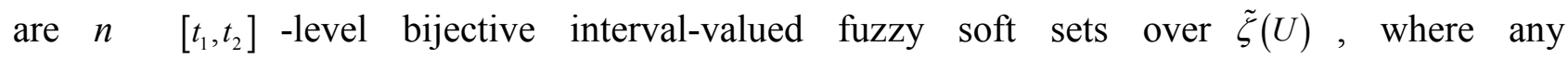
$E_{i} \cap E_{j}=\varnothing(i=1,2,3, \ldots, n ; j=1,2,3, \ldots n ; i \neq j) .(\tilde{\zeta}, B)$ is a $\left[t_{1}, t_{2}\right]$-level bijective interval-valued fuzzy soft set over a common universe $U, B \cap E_{i}=\varnothing(i=1,2,3, \ldots, n)$, and we call it the decision soft set. Suppose $(\tilde{\wp}, E)=\tilde{\cup}_{i=1}^{n}\left(\tilde{\wp}_{i}, E_{i}\right)$.The triple $((\tilde{\wp}, E),(\tilde{\zeta}, B), U)_{\left[t_{1}, t_{2}\right]}$ is called $\left[t_{1}, t_{2}\right]$-level bijective fuzzy soft decision system over a common universe $U$.

In Example 4, we can consider a $\left[t_{1}, t_{2}\right]$-level bijective fuzzy soft decision system $\left(\tilde{\cup}_{i=1}^{3}\left(\tilde{\wp}_{i}, E_{i}\right),\left(\tilde{\wp}_{4}, E_{4}\right), U\right)_{[0.7,08]}$. This bijective fuzzy soft decision system set describes the profit ability of stores and other information.

Definition 13 (bijective interval-valued fuzzy soft decision system dependency) Let $((\tilde{\wp}, E),(\tilde{\zeta}, B), U)_{\left[t_{1}, t_{2}\right]}$ be a $\left[t_{1}, t_{2}\right]$-level bijective fuzzy soft decision system, where $(\tilde{\wp}, E)=\tilde{\cup}_{i=1}^{n}\left(\tilde{\wp}_{i}, E_{i}\right)$ and $\left(\tilde{\wp}_{i}, E_{i}\right)$ is $\left[t_{1}, t_{2}\right]$-level bijective interval-valued fuzzy soft set. $(\tilde{\wp}, E)$ is called condition soft set. The $\left[t_{1}, t_{2}\right]$-level bijective fuzzy soft dependency between $\left(\tilde{\wp}_{1}, E_{1}\right) \wedge\left(\tilde{\wp}_{2}, E_{2}\right) \wedge \ldots \wedge\left(\tilde{\wp}_{n}, E_{n}\right)$ and $(\tilde{\zeta}, B)$ is called $\left[t_{1}, t_{2}\right]$-level bijective fuzzy soft decision system dependency of $((\tilde{\wp}, E),(\tilde{\zeta}, B), U)_{\left[t_{1}, t_{2}\right]}$, denoted by $\kappa_{\left[t_{1}, t_{2}\right]}$ and defined by $\kappa_{\left[t_{1}, t_{2}\right]}=\gamma_{\left[t_{1}, t_{2}\right]}\left(\wedge_{i=1}^{n}\left(\tilde{\wp}_{i}, E_{i}\right),(\tilde{\zeta}, B)\right)$.

Theorem 3 Let $((\tilde{\wp}, E),(\tilde{\zeta}, B), U)_{\left[t_{1}, t_{2}\right]}$ be a $\left[t_{1}, t_{2}\right]$-level bijective fuzzy soft decision system, where $(\tilde{\wp}, E)=\tilde{\cup}_{i=1}^{n}\left(\tilde{\wp}_{i}, E_{i}\right)$ and $\left(\tilde{\wp}_{i}, E_{i}\right)$ is $\left[t_{1}, t_{2}\right]$-level bijective interval-valued fuzzy soft set. $\kappa_{\left[t_{1}, t_{2}\right]}$ is the bijective fuzzy soft decision system dependency of $((\tilde{\wp}, E),(\tilde{\zeta}, B), U)_{\left[t_{1}, t_{2}\right]}$. The dependency between $\wedge_{i=1}^{m}\left(\tilde{\wp}_{i}, E_{i}\right)$, where $m \leq n$, and $(\tilde{\zeta}, B)$ is $\gamma_{\left[t_{1}, t_{2}\right]}\left(\wedge_{i=1}^{m}\left(F_{i}, E_{i}\right),(\tilde{\zeta}, B)\right)$. And $\gamma_{\left[t_{1}, t_{2}\right]}\left(\wedge_{i=1}^{m}\left(\tilde{\wp}_{i}, E_{i}\right),(\tilde{\zeta}, B)\right) \leq \kappa_{\left[t_{1}, t_{2}\right]}$.

In other words, the condition soft set can explain the most detailed classification of decision interval-valued fuzzy soft sets. And removing some bijective interval-valued fuzzy soft sets, it may lose some information of the decision interval-valued fuzzy soft set.

Definition 14 (Reduction of bijective fuzzy soft decision system) Let $((\tilde{\wp}, E),(\tilde{\zeta}, B), U)_{\left[t_{1}, t_{2}\right]}$ be a $\left[t_{1}, t_{2}\right]$ -level bijective fuzzy soft decision system, where $(\tilde{\wp}, E)=\tilde{\cup}_{i=1}^{n}\left(\tilde{\wp}_{i}, E_{i}\right)$ and $\left(\tilde{\wp}_{i}, E_{i}\right)$ is $\left[t_{1}, t_{2}\right]$-level fuzzy bijective soft set, $\cup_{i=1}^{m}\left(\tilde{\wp}_{i}, E_{i}\right) \tilde{\subset}(\tilde{\wp}, E) . \kappa_{\left[t_{1}, t_{2}\right]}$ is the bijective fuzzy soft decision system dependency of $((\tilde{\wp}, E),(\tilde{\zeta}, B), U)_{\left[t_{1}, t_{2}\right]}$ If $\gamma_{\left[t_{1}, t_{2}\right]}\left(\wedge_{i=1}^{m}\left(\tilde{\wp}_{i}, E_{i}\right),(\tilde{\zeta}, B)\right)=\kappa_{\left[t_{1}, t_{2}\right]}$ we say $\cup_{i=1}^{m}\left(\tilde{\wp}_{i}, E_{i}\right)$ is a reduct of a $\left[t_{1}, t_{2}\right]-l e v e l$ bijective fuzzy soft decision system $((\tilde{\wp}, E),(\tilde{\zeta}, B), U)_{\left[t_{1}, t_{2}\right]}$.

Definition 15 (Significance of bijective interval-valued fuzzy soft sets to decision bijective interval-valued fuzzy soft set) Suppose that $\left(\tilde{\cup}_{i=1}^{n}\left(\tilde{\wp}_{i}, E_{i}\right),(\tilde{\zeta}, B), U\right)_{\left[t_{1}, t_{2}\right]}$ is a $\left[t_{1}, t_{2}\right]$-level bijective fuzzy soft decision system. The significance of bijective interval-valued fuzzy soft set to decision bijective interval-valued fuzzy soft set, denoted $\sigma_{\left[t_{1}, t_{2}\right]}\left(\left(\tilde{\wp}_{j}, E_{j}\right), \tilde{\cup}_{i=1}^{n}\left(\tilde{\wp}_{i}, E_{i}\right),(\tilde{\zeta}, B)\right)$, is defined as $\sigma_{\left[t_{1}, t_{2}\right]}\left(\left(\tilde{\wp}_{j}, E_{j}\right), \tilde{\cup}_{i=1}^{n}\left(\tilde{\wp}_{i}, E_{i}\right),(\tilde{\zeta}, B)\right)=\kappa_{\left[t_{1}, t_{2}\right]}-\gamma_{\left[t_{1}, t_{2}\right]}((\tilde{\zeta}, C),(\tilde{\zeta}, B))$, where $(\tilde{\zeta}, C)=\wedge_{i=1}^{n}\left(\tilde{\wp}_{i}, E_{i}\right)(i \neq j)$.

Definition 16 (Core bijective interval-valued fuzzy soft set of decision soft set) Suppose that the $\left[t_{1}, t_{2}\right]$-level bijective interval-valued fuzzy soft set $(\tilde{\varsigma}, C)$ belong to every reduct of $\left[t_{1}, t_{2}\right]$-level bijective fuzzy soft decision system $((\tilde{\wp}, E),(\tilde{\zeta}, B), U)_{\left[t_{1}, t_{2}\right]}$. We say $(\tilde{\zeta}, C)$ is a core $\left[t_{1}, t_{2}\right]$-level bijective interval-valued fuzzy soft set of $((\tilde{\wp}, E),(\tilde{\zeta}, B), U)_{\left[t_{1}, t_{2}\right]}$. 
Definition 17 (Decision rules in bijective fuzzy soft decision system) Let $((\tilde{\wp}, E),(\tilde{\zeta}, B), U)_{\left[t_{1}, t_{2}\right]}$ be a $\left[t_{1}, t_{2}\right]$-level bijective fuzzy soft decision system, where $(\tilde{\wp}, E)=\tilde{\cup}_{i=1}^{n}\left(\tilde{\wp}_{i}, E_{i}\right)$ and $\left(\tilde{\wp}_{i}, E_{i}\right)$ is a $\left[t_{1}, t_{2}\right]$ level bijective interval-valued fuzzy soft set, $\cup_{i=1}^{m}\left(\tilde{\wp}_{i}, E_{i}\right) \tilde{\subset}(\tilde{\wp}, E)$ is a reduct of $\left[t_{1}, t_{2}\right]$-level bijective fuzzy soft decision system $((\tilde{\wp}, E),(\tilde{\zeta}, B), U)_{\left[t_{1}, t_{2}\right]}$. Suppose that $(\varsigma, C)=\wedge_{i=1}^{m}\left(\wp_{i}, E_{i}\right)$. We call if $e_{i}$ then $e_{j}($ $\left.\tilde{T}\left(e_{i}\right)_{\left[t_{1}, t_{2}\right]} / \tilde{T}\left(e_{j}\right)_{\left[t_{1}, t_{2}\right]}\right)$ a decision rule induced by $\cup_{i=1}^{m}\left(\tilde{\wp}_{i}, E_{i}\right)$, where $e_{i} \in C$ and $\tilde{\zeta}\left(e_{j}\right) \supseteq \tilde{\varsigma}\left(e_{i}\right)$ and $e_{j} \in B$ and $\tilde{T}\left(e_{i}\right)_{\left[t_{1}, t_{2}\right]} / \tilde{T}\left(e_{j}\right)_{\left[t_{1}, t_{2}\right]}$ denotes the coverage proportion of rule. .

\section{Conclusion}

This paper proposed the concept of bijective interval-valued fuzzy soft set and described the restricted $A N D$ operation of it, based on $\left[t_{1}, t_{2}\right]$-level soft sets. Afterwards, we defined a bijective interval-valued fuzzy soft decision system, and studied some correspondent data mining techniques, such as dependency, reduction and decision rules.

\section{Acknowledgements}

Our work is sponsored by Science and Technology Research Project of Chongqing Municipal Education Commission (KJ110414), National Science Foundation Project of CQ CSTC(cstcjjA30007), Humanity and Social Science Youth foundation of Ministry of Education of China (12YJC630053).

\section{References}

[1] Molodtsov, D., Soft Set Theory First Results. Computers \& Mathematics With Applications, 1999. 37(4-5): p. 19-31.

[2] Zou, Y. and Z. Xiao, Data analysis approaches of soft sets under incomplete information. Knowledge-Based Systems, 2008. 21(8): p. 941-945.

[3] Maji, P.K., A.R. Roy and R. Biswas, an application of soft sets in a decision making problem. Computers \& Mathematics with Applications, 2002. 44(8): p. 1077-1083.

[4] Çagman, N. and S. Enginoglu, Soft set theory and uni-int decision making. European Journal of Operational Research, 2010. 207(2): p. 848-855.

[5] Kong, Z., L. Gao, L. Wang, and S. Li, The normal parameter reduction of soft sets and its algorithm. Computers \& Mathematics with Applications, 2008. 56(12): p. 3029-3037.

[6] Gong, K., Z. Xiao and X. Zhang, The bijective soft set with its operations. Computers \& Mathematics with Applications, 2010. 60(8): p. 2270-2278.

[7] Yang, X., T.Y. Lin, J. Yang, Y. Li, and D. Yu, Combination of interval-valued fuzzy set and soft set. Computers \& Mathematics with Applications, 2009. 58(3): 521-527.

[8] Roy, A.R. and P.K. Maji, A fuzzy soft set theoretic approach to decision making problems. Journal of Computational and Applied Mathematics, 2007. 203(2): p. 412-418.

[9] F. Feng, Y.B. Jun, X.Y. Liu, L.F. Li, An adjustable approach to fuzzy soft set based decision making, J. Comput. Appl. Math. 2010.234(1):p.10-20. 